\title{
THE INTERNAL GONSTITUTION OF SUNSPOTS
}

\author{
A. SCHLÜTER AND S. TEMESVÁRY \\ Max-Planck-Institut für Physik, Göttingen, Germany
}

\begin{abstract}
ABSTRAGT
The constitution of stationary single sunspots of circular shape is considered. Account is taken of the mechanical effects of the magnetic field, including those which arise from the curvature of the lines of force. To make the system of magneto-hydrostatic equations manageable, it is assumed that the relative distribution of the vertical component of the magnetic field is the same across the flux-tube of the spot in all depths. Preliminary results indicate that suppression of convective energy transport by the magnetic field in those depths in which ionization of hydrogen takes place, will give the essential observable properties of sunspots, relatively independent on the asumptions about the physical processes in greater depths. There the physical properties of matter can deviate but very little from those of the indisturbed hydrogen convection zone.
\end{abstract}

\section{ASSUMPTIONS}

The assumptions made in this attempt to determine the relation between the constitution of sunspots and their magnetic field divide into two groups; those which seem obvious, if we deal with a simple picture of a sunspot (group A), and those which determine the particular model to be considered (group B). The latter are not necessarily true, but are suggested for a first attempt by their simplicity.

A I. We consider a circularly shaped single spot $\left(\mathrm{H}_{\mathrm{I}}\right.$ in the Zurich classification). Therefore cylindrical symmetry around the $z$-axis of a co-ordinate system $r, \phi, z$ is assumed, the $z$-axis being perpendicular to the sun's surface, which is taken to be plane, the positive direction of $z$ pointing toward the centre of the sun. On the axis (i.e. for $r=0$ ) the electric current and the $r$-component of the field $H_{r}$ vanish, and the vertical component $H_{z}$ has there a maximum.

A 2. The spot is assumed to be in a quasi-static equilibrium, that means, all derivatives with respect to time are put equal to zero. This seems a fair approximation for relatively long-living single spots. 
A 3. The magnetic field strength and its derivatives vanish for $z=-\infty$ (infinite distance above the sun) and for $r=\infty$.

$B_{\text {I. }}$ All material motions in the spot are neglected.

B 2. The magnetic field has no torsion, that means $H_{\phi}$ vanishes everywhere, each line of force lying in a plane perpendicular to the sun's surface.

B 3. We assume that the relative distributon of magnetic flux through a horizontal cross-section of the sunspot is everywhere geometrically similar.

\section{FORMULATION OF THE SIMILARITY-ASSUMPTION}

The ratio of the vertical component $H_{z}(z, r)$ to the central intensity $H(z)$ at the same depth $z$ is assumed to depend on $z$ only by a scale factor $\zeta(z)$, or

$$
H_{z}(z, r)=H(z) . D(\alpha) / D(0) ; \quad \alpha=\zeta(z) . r .
$$

Then $\mathrm{I} / \zeta(z)$ describes the dependence on depth of the diameter of a fluxtube constituting the magnetic field of the sunspot, and the function $D(\alpha)$ determines the shape. Due to the continuity of the lines of force (i.e. $\operatorname{div} \mathbf{H}=0) H_{z}$ at homologous points (i.e. $\alpha$ fixed) and $H(z)=H_{z}(z, 0)$ in particular, have to be larger where the tube is more constricted and vice versa, they vary in fact like $\zeta^{2}(z)$, so that with a suitable normalization of $D$ we may write for the field on the axis

$$
H(z)=\zeta^{2}(z) . D(0)
$$

and generally for the vertical component

$$
H_{z}(z, r)=\zeta^{2}(z) . D(\alpha) ; \quad \alpha=\zeta(z) . r .
$$

Using once more the equation $\operatorname{div} \mathbf{H}=0$, we find the component of the field perpendicular to the axis of the field-tube to be given by:

$$
H_{r}(z, r)=-(d \zeta / d z) \cdot \alpha D(\alpha) .
$$

The condition of similarity of flux-distribution therefore implies that the ratio of the field components increases linearly with the distance from the axis

$$
\frac{H_{r}}{H_{z}}=-r \frac{d \ln \zeta}{d z}
$$

The quantity $\alpha$ introduced so far as the distance from the axis scaled down by $\zeta$, has a simple meaning: The equation

$$
\begin{gathered}
\alpha(\zeta, r)=\text { const } \\
264
\end{gathered}
$$


determines the lines of force, i.e. $\alpha$ is constant along the lines of force. For the magnetic flux through a horizontal circle of radius $r$ we obtain

$$
2 \pi \int_{0}^{r} H_{z} r d r=2 \pi \int_{0}^{\alpha} D(\alpha) d \alpha .
$$

The magnetic flux between a given line of force and the axis is therefore independent of depth, as it has to be.

\section{THE DIFFERENTIAL EQUATION FOR THE MAGNETO-HYDROSTATIC EQUILIBRIUM}

According to the assumptions ( $\mathrm{A}_{\mathrm{I}}$ ) and (B I) we have

$$
\frac{\mathbf{I}}{4 \pi} \mathbf{H} . \operatorname{curl} \mathbf{H}=\rho \mathbf{g}-\operatorname{grad} p,
$$

$\rho$ being the density, $\mathbf{g}$ the gravitational acceleration and $p$ the gas pressure. The $r$-component of $(7)$ is

$$
H_{z}\left(\frac{\partial H_{r}}{\partial z}-\frac{\partial H_{z}}{\partial r}\right)=4 \pi \frac{\partial p}{\partial r}
$$

or using a dash to denote the derivative with respect to the depth $z$

$$
\alpha D^{2} \zeta \zeta^{\prime \prime}+\frac{\mathrm{I}}{2} \frac{d}{d \alpha}(\alpha D)^{2} \cdot \zeta^{\prime 2}+\frac{\mathrm{I}}{2} \frac{d}{d \alpha}\left(D^{2}\right) \cdot \zeta^{4}=-4 \pi \frac{d p}{d \alpha} .
$$

This gives, integrated over $r$ (that is over $\alpha$ ) from the axis to infinity,

$$
\zeta \zeta^{\prime \prime} \int_{0}^{\infty} \alpha D^{2}(\alpha) d \alpha-\frac{1}{2} \zeta^{4} D^{2}(\mathrm{o})=-4 \pi \Delta p
$$

because $\alpha D$ vanishes for $\alpha=0$ as well as for $\alpha=\infty$, with

and the abbreviations

$$
\Delta p=p(\infty, z)-p(0, z) \text {, }
$$

$$
H=\zeta^{2} D(\mathrm{o})=y^{2} \text { and } f=\frac{2}{D(0)} \int_{0}^{\infty} \alpha D^{2}(\alpha) d \alpha
$$

we obtain

$$
f y y^{\prime \prime}-y^{4}+8 \pi \Delta p=0 \text {; }
$$

the basic differential equation of the magnetic field.

The $z$-component of (7) gives for the pressure difference between the axis and the undisturbed layers of the same depth:

$$
(\Delta p)^{\prime}=g \Delta \rho,
$$

(where $\Delta \rho=\rho(\infty, z)-\rho(0, z)$ ) because the magnetic force vanishes for $r=0$ and $r=\infty$. Due to our assumption of constancy of shape we need only to know the physical state of the matter along the axis of the field-tube 
and in great distances from this tube and our partial differential equation (7) reduces to an ordinary one with the depth as the only independent variable. The term $y^{4}$ in Eq. (9) represents the effect of the magnetic pressure across the lines of force, while the term $f y y^{\prime \prime}$ expresses the effect of the Maxwell-Faraday tension along the lines of force, which comes into play only where these lines are curved. The usual neglect of this term is inadmissible, particularly above the photosphere where $\Delta p$ certainly becomes insignificantly small. Before entering into the more complete discussion of the constitution of the field-tube we consider some formal properties of Eq. (9).

$y^{\prime \prime}=0$ is a solution of this equation. if

$$
y^{4} / 8 \pi=H^{2} / 8 \pi=\Delta p
$$

and at the same time

$$
y^{\prime}=\text { const }=a \text {, }
$$

say, that gives

$$
\left.\begin{array}{rl}
y & =a\left(z-z_{0}\right), \\
8 \pi \Delta p & =a^{4}\left(z-z_{0}\right)^{4}, \\
2 \pi g \Delta \rho & =a^{4}\left(z-z_{0}\right)^{3},
\end{array}\right\} \quad a \neq 0 .
$$

It is interesting to note that configurations can exist, where the tension along the lines of force do not contribute to the lateral forces even though the lines of force are not straight (i.e. $a \neq 0$ ); the practical importance of these configurations must however be quite limited since they possess an essential singularity at the point $z=z_{0}$, where the diameter of the flux-tube $(\alpha \mathrm{I} / y)$ becomes infinite. Yet, the case $a=0$ with $\Delta p=$ const; $\Delta \rho=0$ may be a good approximation wherever the flux-tube is sufficiently slender, this is, however, at least near the photosphere, certainly no good approximation.

If $y^{\prime}, y \neq 0$ we may multiply Eq. (9) by $y^{\prime} / y$ and integrate once to obtain

$$
\begin{aligned}
f y^{\prime 2} & =\frac{1}{2} y^{4}-16 \pi \int \Delta p \frac{y^{\prime}}{y} d z+\text { const } \\
& =\frac{1}{2} y^{4}+16 \pi g \int \Delta \rho \ln y d z+\text { const. }
\end{aligned}
$$

This form shows that above the sun's surface $(z<0)$ where to a very good approximation $\Delta p=0$, the condition of vanishing field strength for infinite distance leads to the first-order equation

$$
f y^{\prime 2}=\frac{1}{2} y^{4}
$$

The solutions of (9) for the interior $(z>0)$ have to be adapted to this 266 
condition. The only solutions of (15) which are regular for all $z<0$ are of the form

$$
H=y^{2}=\frac{2 f}{\left(z-z_{0}\right)^{2}}
$$

where $z_{0}>0$ is the point in which the field strength would become infinite, if the solution were continued into the interior. The equation of the lines of force is

$$
r=\text { const }\left(z-z_{0}\right) \text {. }
$$

This is the equation of straight lines which intersect the axis $r=0$ at $z_{0}$. The field therefore falls off like that of a monopole, which is an obvious consequence of our assumptions.

\section{PARTIGULAR DISTRIBUTIONS OF \\ MAGNETIC FLUX}

By the Eqs. (6) and (8) the constant $f$ is related to the total magnetic flux $F$ by:

$$
\pi f \mid F=\int_{0}^{\infty} \alpha D^{2}(\alpha) d \alpha / D(\mathrm{o}) \int_{0}^{\infty} \alpha D(\alpha) d \alpha .
$$

We study this relation by considering some examples for the shape-function $D(\alpha)$. We remark, that this quotient is invariant against the substitution $c_{1} D\left(c_{2} \alpha\right)$ for $D(\alpha), c_{1}$ and $c_{2}$ being constants.

I. If we assume a gaussian shape for the horizontal distribution of magnetic flux, namely

we obtain by Eq. (18)

$$
\begin{gathered}
D(\alpha)=D(0) e^{-\alpha^{2}}, \\
F=2 \pi f .
\end{gathered}
$$

2. The observations of flux-distribution at the surface of sunspots lead to the well-known Broxon formula, which gives

$$
H_{z}=H_{0}\left(\mathrm{I}-\frac{r^{2}}{r_{0}^{2}}\right) \cos \left(\frac{\pi}{2} \frac{r}{r_{0}}\right),
$$

where $H_{0}$ is the central field strength and $r_{0}$ the radius of the penumbra, and further very closely

$$
F=2 \pi \int_{0}^{\infty} H_{z} r d r \approx H_{0} r_{0}^{2}
$$

$$
\begin{aligned}
& \text { Putting } \\
& \quad \alpha=\frac{\pi}{2} \frac{r}{r_{0}}, \\
& \text { we obtain } \quad D(\alpha)=D(0)\left(\mathrm{I}-\frac{4}{\pi^{2}} \alpha^{2}\right) \cos \alpha \text { for } \alpha<\frac{\pi}{2}
\end{aligned}
$$


and

$$
D(\alpha)=0 \text { for } \alpha \geqslant \frac{\pi}{2} \text {. }
$$

So we obtain from Eq. (18) $\quad F=\mathrm{I} \cdot 70 \pi f$.

Considering the uncertainty of the Broxon formula (cf. W. Mattig [1] and G. Thiessen [2]) the difference between Eqs. (20) and (25) seems to be unimportant.

3. We have assumed $H_{z}$ to have everywhere the same sign and have neglected the returning lines of force, while it is more probable that in reality the total magnetic flux must equal zero. To estimate the influence of these returning lines of force we assume that we have two concentric tubes, both with constant $H_{z}$, the outer tube of $k$-fold radius representing the (unobserved) returning lines of force. That means:

$$
D(\alpha)=\left\{\begin{array}{lll}
D(\mathrm{o}) & \text { for } & 0 \leqslant \alpha<\mathrm{I}, \\
-D(\mathrm{o}) /\left(k^{2}-\mathrm{I}\right) & \text { for } & \mathrm{I} \leqslant \alpha<k \\
0 & \text { for } & k \leqslant \alpha .
\end{array}\right.
$$

The observed flux has then to be taken as the flux $F(\mathrm{r})$ of the inner tube $(\alpha<\mathrm{I})$ for which we get

$$
F(\mathrm{I})=\left(\mathrm{I}-\frac{\mathrm{I}}{k^{2}}\right) \pi f
$$

The deviation due to the returning lines is expressed by the term with $\mathrm{I} / k^{2}$ and will be of the order of a few per cent, if $k$ is of the order Io or more.

The examples given show the relation between $f$ and the flux-distribution. If we want to apply this to the field of actual sunspots, we have first to test the applicability of the underlying assumption of similarity. We recall, that we have no a priori reason as to why this similarity should hold, other than this being the simplest possibility. We can test this hypothesis only at the surface, where both $H_{z}$ and $H_{r}$ are observable. According to Eq. (5) the ratio of $H_{r} / H_{z}$ should theoretically increase linearly with $r$. The observations are usually quoted as showing this ratio to vary like $\operatorname{tg}\left(\pi r / 2 r_{0}\right)$ where $r_{0}=$ radius of the sunspot. Fig. I (adapted from S. Chapman [3]) shows, however, that the observations are as well compatible with a linear increase as with the quoted relation. Assuming now the linear law to hold sufficiently well, also the increase with depth of the field on the axis follows from Eq. (5). It results in

$$
\begin{aligned}
d H / d z & \approx 4 H / r_{0} \\
& \approx 0.8 \text { gauss } / \mathrm{km}
\end{aligned}
$$

(with $H \approx 2,000$ gauss; $r_{0} \approx 10,000 \mathrm{~km}$ ) in fair agreement with the value 0.5 gauss $/ \mathrm{km}$ found previously by Houtgast and van Sluiters [4]. 


\section{THE ENERGY TRANSPORT IN THE SPOT}

The problem remains of determining the function $\Delta p(z)$. We obtain a necessary condition on $\Delta p$ by integrating the basic differential Eq. (9) over $z$, between $z_{1}$ and $z_{2}$, say:

$$
\int_{z_{1}}^{z_{2}}\left(f y^{\prime 2}+y^{4}\right) d z+\frac{f}{2}\left\{y^{2^{\prime}}\left(z_{1}\right)-y^{2^{\prime}}\left(z_{2}\right)\right\}=8 \pi \int_{z_{1}}^{z_{2}} \Delta p d z
$$

If we choose for $z_{1}$ a sufficiently great height above the sun, then $y^{2 \prime}\left(z_{1}\right)$ disappears. So does $y^{2 \prime}\left(z_{2}\right)$ if we choose such a great depth $z_{2}$ that the field

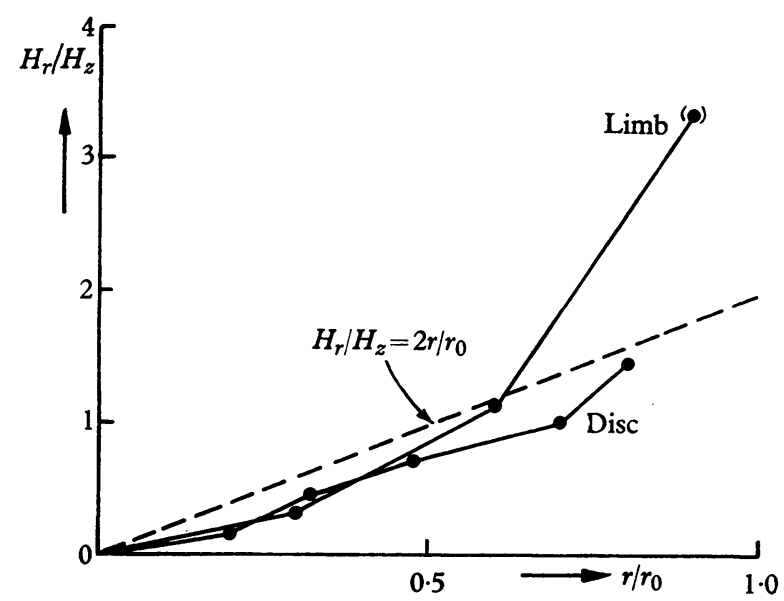

Fig. 1 . The ratio between the radial and vertical components of the magnetic field in a sunspot plotted as a function of $r / r_{0}$. Full curves indicate observed values.

either is constant, or has disappeared there. It then follows that the mean value of $\Delta p$ must be positive and must even be larger than would follow by just considering the 'magnetic pressure' $H^{2} / 8 \pi=y^{4} / 8 \pi$.

On the axis, hydrostatic equilibrium is not affected by the magnetic field, nor is, of course, the equation of state. Therefore the only possibility is that the temperature is different, and since the temperature is controlled by the transport of energy, this transport must be affected by the presence of the magnetic field of the spot. This can conceivably be the case where the energy is ordinarily transported not by radiation but by convection. So one is lead to the picture first proposed by L. Biermann [5]: The convective transport of energy in the hydrogen convection zone is hindered by the magnetic field of the sunspot. This causes the temperature to drop faster in the outward direction, thereby producing a positive $\Delta p$, 
which then is needed to keep the lines of force together and so to make the existence of the sunspot field possible. At the same time the reduction of the effective thermal conductivity deflects a part of the energy flow out of the sunspot-tube, thus causing the lowering of the effective temperature at the surface.

Since, as yet, a theory of the influence of a magnetic field on convection and energy-transport in a zone of great thermal instability does not exist, the effect of the sunspot-field in the hydrogen convection zone cannot be asserted with sufficient certainty. One may, however, surmise that a necessary condition for convection to be effectively suppressed is gained by using the scale height as the characteristic length in Walén's stability criterion [6], which then reads:

Stability occurs if

$$
\left(\frac{d \ln T}{d \ln p}\right)_{\text {radiative }}-\frac{H^{2}}{4 \pi p}<\left(\frac{\mathrm{d} \ln T}{d \ln p}\right)_{\text {adiabatic }} .
$$

\section{NUMERIGAL INTEGRATIONS}

Lacking safe theoretical guidance, one has now to make an assumption about the energy transport below the sunspot, then one has to evaluate $\Delta p$ by numerical integration and to solve the differential Eq. (9) for the magnetic field. Since this part of the work is still in progress, we shall give only a brief sketch of our attempts.

I. First we supposed that the suppression of convection inside the spottube is complete and that the energy is there transported by radiation and in vertical direction only. With these assumptions the temperature increases with depth much faster inside the tube than outside it, particularly so in the hydrogen-ionization zone. With the so determined $\Delta p$ we tried to find solutions for the magnetic field. However, it turned out, that with no reasonable assumptions on $y$ and $y^{\prime}$ at the surface a solution for $y(z)$ existed which did not become irregular within a depth of a few scale heights. From these numerical attempts it followed that at least in greater depths the pressure difference $\Delta p$ must be very small compared to $p$ for a regular solution of Eq. (9) to exist.

2. To gain an impression what $\Delta p$ should look like, we then assumed a reasonable shape for the vertical dependence of the magnetic field and determined by Eq. (9) $\Delta p$ and thereby $\Delta \rho$ and $\Delta T$. We so circumvented the difficulty of determining the energy transport mechanism. Two models were considered in particular. 
(i)

$$
\begin{gathered}
H=y^{2}=2 f d^{-2}\left[\ln \left\{\exp \left(\frac{\mathrm{I}}{d} \sqrt{ }\left(2 f / H_{c}\right)\right)+\exp \left(-\frac{z-z_{0}}{d}\right)\right\}\right]^{-2}, \\
f, d, H_{c}, z_{0}=\text { const. }
\end{gathered}
$$

For $z \rightarrow+\infty$ the field becomes constant $\left(H \rightarrow H_{c}\right)$, and for $z \rightarrow-\infty$ the solution ( 16 ) is approached.

$$
\begin{gathered}
H=y^{2}=\frac{2 f}{a^{2}+\left(z-z_{0}\right)^{2}}, \\
a, z_{0}=\text { const. }
\end{gathered}
$$

Here the magnetic field drops off also as $z$ goes to $+\infty$. The pressure difference is given by

$$
4 \pi \Delta p=\frac{3 a^{2} f^{2}}{\left[a^{2}+\left(z-z_{0}\right)^{2}\right]^{3}}
$$

and falls rapidly with increasing distance from the point of maximal field strength $z=z_{0}$, so that the solution ( $\mathrm{I} 6$ ) is approximated.

A number of plausible parameters were tried in these models. In every case, it turned out that in a depth well within the hydrogen convection zone the internal constitution of the flux-tube was almost indistinguishable from that of the undisturbed layers.

3. The first attempts have shown, that some modification has to be made in the assumption of pure radiative transport of the observed energy flux in the spot-tube. The easiest explanation would be that the suppression of convective energy transport is far from being complete. Since, however, every detailed assumption on the effectiveness of the convective transport mechanism under the conditions prevailing in the spot-tube would be completely arbitrary, we considered an alternative solution. In addition, the stability criterion (28) showed no reason for instability in the examples considered by us.

If the magnetic field is much stronger in great depths than it is near the surface, then the flux-tube is there correspondingly thinner and horizontal flow of energy may become appreciable. This might be a mechanism which causes the desired similarity in the thermodynamic state of the gas in the tube and of the gas outside it. To obtain a model containing the horizontal energy transport, we assume that the temperature across the flux-tube is given by a gaussian distribution the half-width of which corresponds to the diameter of the flux-tube

$$
T(r, z)=T(\infty, z)-\Delta T(z) \exp \left(-\frac{y^{2}(z) r^{2}}{2 f}\right)
$$


Since we assume radiative equilibrium we have the equations:

$$
\begin{gathered}
\operatorname{div} \mathbf{F}=0, \quad \mathbf{F}=\text { radiative energy flux, } \\
\mathbf{F}=-\sigma \operatorname{grad} T, \quad \sigma=\text { thermal conductivity (due } \\
\text { to radiative transport). }
\end{gathered}
$$

Similar as before we fulfil these equations only on the axis $(r=0)$, taking $\sigma$ and the molecular weight $\mu$ to depend only on $z$ (not on $r$ ). So we arrive at the following system:

$$
\left.\begin{array}{rl}
f y y^{\prime \prime} & =y^{4}-8 \pi \Delta p, \\
(\Delta p)^{\prime} & =g \Delta \rho, \\
(\Delta T)^{\prime} & =\sigma^{-1} \Delta F, \\
(\Delta F)^{\prime} & =\frac{2}{f} \sigma y^{2} \Delta T+F^{\prime}(\infty, z),
\end{array}\right\}
$$

where all quantities (except the constants $g$ and $f$ ) depend on depth only. $F(\infty, z)$ denotes the part of the energy transported vertically by radiation in the undisturbed layers.

These equations, together with the equation of state and suitable boundary conditions suffice to determine the magnetic field (i.e. $y(z))$ and the difference in constitution between the flux-tube and the normal layers of the sun. If one starts at the surface (at $z=0$, say), then the values of $y$ and $y^{\prime}$ are provided by the magnetic measurements (together with the value of $f$ ), while the values of $\Delta F$ and $\Delta T$ are determined by the observed defect in brightness of the sunspot. The value of $\Delta \rho$ is not directly observable since it depends essentially on the geometric depression of the spot which is very hard to determine.

For our attempts to solve these equations numerically, we used for the constitution of the undisturbed layers a model, the dates of which were kindly placed at our disposal by Mrs Böhm-Vitense [7].

It turned out, that the solutions of Eq. (35) were unstable to a degree, which we had not anticipated, the range of initial conditions for which the solution could be continued to a depth of more than a few thousand kilometres, being extremely small. This then means, that the condition that the solution should be regular down to a depth where the ionization of hydrogen is complete, determines the constitution of the sunspot for all higher layers, if only $f$ is given. (Only at this depth the horizontal flux of energy becomes important, if at all.) This means that we can not infer anything about the constitution at greater depths, except that we know the difference between the spot and the normal layers to be quite small, as 
we pointed out earlier. These results are to be considered as preliminary, since the work described here is still in progress. In particular, we are attempting to obtain a consistent model for the constitution of the decisive part of the sunspot-tube from the photosphere down to the hydrogen ionization zone.

\section{REFERENGES}

[1] Mattig, W. Z. Astrophys. 31, 273, 1953.

[2] Thiessen, G. Naturwissenschaften, 40, 2 18, 1953.

[3] Chapman, S. Mon. Not. R. Astr. Soc. 103, I I $7,1943$.

[4] Houtgast, J. and van Sluiters, A. B.A.N. 10, 325, 1948.

[5] Biermann, L. Vjschr. Astr. Ges., Lpz. 76, 194, 1941 .

[6] Walén, G. On the Vibratory Rotation of the Sun (Stockholm, 1949), cf. The Sun, ed. G. P. Kuiper (Chicago, I953), ch. 8 (T. G. Cowling), pp. $56 \mathrm{I}-5$.

[7] Vitense, E. Z. Astrophys. 32, I 35, 1953.

\section{Discussion on Papers 28 and 29}

Bostick: One mechanism for the cooling of sunspots is that provided by the magnetic evaporation of hot ions and electrons by the presence at the sunspot of the divergent magnetic field which acts like a magnetic vacuum pump. It can be calculated that a hydrogen ion and electron pair with an energy $W_{\perp}$ greater than $3 \mathrm{eV}$ transverse to the sunspot magnetic field will feel an upward force $\left(W_{\perp} / H\right)$ grad $H$ which is greater than the gravitation force on the ion. Accordingly, the hot ion pairs will be evaporated from the sunspot, leaving the cooler ones behind. This mechanism is expected to be operating strongly in regions where the Larmor frequency of the electrons exceeds the collision frequency. This mechanism can also conceivably explain the penumbra of the sunspot, because we expect the thickness of the penumbra to be representative of the thickness of the current sheet producing the magnetic field of the spot: Within this current sheet grad $H$ in the vertical direction is less and we therefore expect less evaporation cooling in the penumbra. The sharp boundary of the penumbra is presumably produced by the edge of the current sheet where the cooling mechanism disappears completely.

The fact that solar prominences are streamers, frequently from one sunspot to another, suggests that the same mechanism is operative here as in the laboratory (Bostick, W. H., Phys. Rev. 104, I I9I, I956), where we have produced streamers in a geometrically similar magnetic field generated by a horseshoe magnet. The important fact brought forward by the laboratory experiments is that for streamer formation it is necessary to have not only the magnetic field from north to south but to have a current flowing between north and south as well. Hence, we infer that the probable distribution of magnetic field, currents, and velocity in a whirl ring producing the spot pair is helical, i.e. the field lines form helices around the central circle of the ring. This helical distribution of the magnetic field in the ring will cause a current to flow from north to south or vice versa when the ring intersects the surface of the sun (see also page I 13 of this volume). 
Alfvén: This is a very interesting idea you put forward but it is necessary to check in detail whether the similarity transformation from your plasmoid to the conditions in the sun could be made. I look forward with great interest to future results in this field.

Dungey: There is some circumstantial evidence that the sunspot fields are twisted. I wish someone would look for this in the Zeeman effect.

Biermann: I would like to add here one remark which should have been made in my report. A magnetic field of the kind I discussed can be stationary (against drifting towards the surface, owing to smaller density) only if it is approximately of the type called 'force-free' field by Lüst and Schlüter. Hence, it would seem to be worth while to look after screw type geometrical features also of the magnetic spot fields on the surface. 